\title{
Wheeler's delayed-choice gedanken experiment with a single atom
}

\author{
A. G. Manning, R. I. Khakimov, R. G. Dall and A. G. Truscott*
}

The wave-particle dual nature of light and matter and the fact that the choice of measurement determines which one of these two seemingly incompatible behaviours we observe are examples of the counterintuitive features of quantum mechanics. They are illustrated by Wheeler's famous 'delayedchoice' experiment ${ }^{1}$, recently demonstrated in a single-photon experiment ${ }^{2}$. Here, we use a single ultracold metastable helium atom in a Mach-Zehnder interferometer to create an atomic analogue of Wheeler's original proposal. Our experiment confirms Bohr's view that it does not make sense to ascribe the wave or particle behaviour to a massive particle before the measurement takes place'. This result is encouraging for current work towards entanglement and Bell's theorem tests in macroscopic systems of massive particles ${ }^{3}$.

The question of whether light behaves like a particle or wave had a long and strongly contested history until the advent of quantum mechanics, where it was accepted that it could indeed exhibit either behaviour. Conversely, it was de Broglie's hypothesis of matter waves ${ }^{4}$ that deviated from the preceding view of massive bodies exclusively as particles, which was confirmed by the electron diffraction experiments of Davisson and Germer ${ }^{5}$. Even more bizarrely, the way in which an experiment is performed seems to induce one of these behaviours to the exclusion of the other. The question of whether a single photon in an interferometer passes through either one arm (as a particle) or both simultaneously (as a wave) led to Wheeler devising his famous gedanken experiment, which supposed that the decision of whether to attempt to measure particle or wave behaviour is made after the photon enters the interferometer. By removing the second beamsplitter of the interferometer (Fig. 1a), which-way information is revealed ${ }^{6}$, which precludes an interference measurement, while inserting the beamsplitter destroys information about the path taken by the photon and re-establishes a wave interference dependent on the phase difference $\phi$ between the arms.

Although many experiments have shown particle-wave duality with photons ${ }^{7}$, including delayed-choice schemes ${ }^{8-10}$, delayed-choice quantum eraser experiments ${ }^{11}$ and entanglement swapping using delayed choice ${ }^{12}$, only recently has the complete scheme proposed by Wheeler been realized experimentally ${ }^{2}$. By simultaneously ensuring that only a single photon is present in the interferometer at once, and that the decision of interferometer configuration is relativistically separated from the photon's entry to the interferometer, it was unambiguously shown that Wheeler's supposition that such a choice affects the 'past history' of the photon was correct.

Recent advances in the trapping and cooling of atoms has led to the ability to readily observe wavelike phenomena with particles that have mass, such as the interference between two Bose-Einstein condensates ${ }^{13}$. However, progress towards demonstrating Wheeler's experiment with massive particles, such

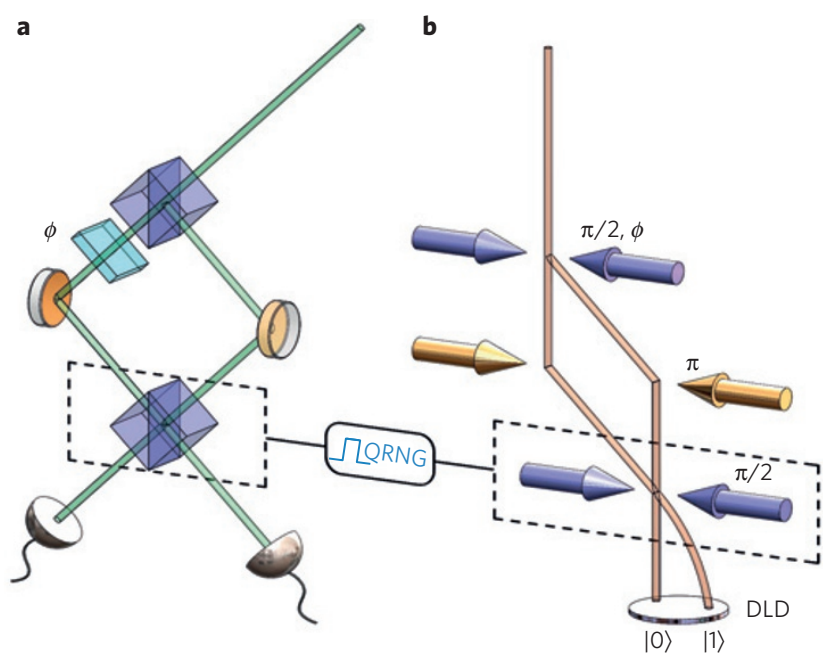

Figure 1 | Schematics of Wheeler's delayed-choice experiments. a, Optical version of Wheeler's delayed-choice experiment. $\mathbf{b}$, Atomic version of Wheeler's experiment, where the physical beamsplitters and mirrors are now replaced with optical Bragg pulses. A quantum random number generator (QRNG) is used to decide whether the last beamsplitting pulse is either implemented or not. The random number is triggered and chosen after the $\pi$-pulse (mirror pulse), thereby ensuring that the atom has no prior knowledge of how it will be detected when it enters the interferometer.

as a delayed-choice Stern-Gerlach interferometer with metastable hydrogen ${ }^{14}$ or a spin interferometer with neutrons ${ }^{15}$, have been limited. Importantly, these experiments used beams containing more than one particle in the apparatus at any one time, rendering a meaningful test of Wheeler's ideas impossible, at least at the quantum level. Nonetheless, they demonstrate an advantage of using massive particles over photons, the relatively slow velocity (compared to light) of the atoms through the interferometer allows an increased time for making the delayed choice.

Here, we report the first realization of Wheelers famous delayedchoice gedanken experiment with single massive particles. The only successful demonstration of Wheeler's ideas so far has been achieved with single photons ${ }^{2}$. Here we use atoms, which is an important distinction, since atoms have many internal degrees of freedom. This allows coupling to the external environment through, for example, the atom's sensitivity to magnetic and electric fields. Moreover, an atom has significant mass, which allows strong coupling to gravitational fields. These interactions of the atom with its environment are required for the appearance of decoherence; thus, in this sense an atom can be thought of as a more classical particle than a photon. As such, our experiment tests Wheeler's ideas in a regime in which it has never been tested. 
We use an ultracold $(<1 \mathrm{nK})$ source of single atoms ${ }^{16}$ to implement Wheeler's idea: that the behaviour of the atom can indeed be induced by our choice of measurement. Our experimental setup closely mirrors that of the Mach-Zehnder scheme originally proposed by Wheeler (Fig. 1b), where essentially the roles of light and matter have been reversed. Atoms are released from an optical dipole trap (see Methods) and fall under gravity towards a delayline detector (DLD) capable of imaging single $\mathrm{He}^{*}$ atoms with threedimensional information.

About $1 \mathrm{~ms}$ after releasing the atoms from the trap, a pair of laser beams are used to Bragg scatter the atoms into different momentum modes (see Methods) ${ }^{17}$, where for a Rabi frequency $\Omega$, a Bragg pulse of duration $t_{\pi}=\pi / \Omega$ coherently transfers from one momentum mode to another in a two-state system ${ }^{18}$, and a pulse duration $t_{\pi / 2}=\pi /(2 \Omega)$ transfers one momentum mode into an equal superposition of the two modes. The action of a $\pi$ pulse is thus analogous to a mirror, while the $\pi / 2$ pulse acts as a 50:50 beamsplitter. An arbitrary phase can be applied to the atomic wavefunction by controlling the phase of the Bragg pulses. In particular, we set the phase to zero for all pulses except for the first $\pi / 2$ pulse, which carries an adjustable relative phase $\phi$.

After applying the $\pi$ pulse, a quantum random bit generator (see Methods) is triggered to decide whether the second $\pi / 2$ pulse should be initiated, which is equivalent to the addition or removal of the second beamsplitter in the optical case (Fig. 1a). In our experiment, we use Bragg pulses of order $20 \mu$ s (and a $\pi / 2-\pi-\pi / 2$ sequence of $200 \mu$ s duration) and generate the random bit and implement its result $170 \mu$ s after the first beamsplitting pulse-thus ensuring that the choice of detection is made well after the atom passes the first beamsplitter and also that the atom has no prior knowledge about the final configuration of the interferometer.

If the second $\pi / 2$ pulse is applied, then the arms of the interferometer are mixed, and the relative phase between the arms $\phi$, generated at the first beamsplitter, can affect the probability of each atom being in a given momentum mode. Should this pulse be disabled, then there is a $50 \%$ chance that the atom will travel down either path of the interferometer in the 'open' configuration, which means that the average count rate for each momentum mode will be $50 \%$ irrespective of the phase $\phi$ applied. Instead of having a separate detector to measure counts in each arm of the interferometer, we can easily resolve the two momentum modes due to their arrival at different spatial positions and times on the same DLD (ref. 19; see Fig. 2).

It is interesting to contrast the photon demonstration of Wheeler's experiment ${ }^{2}$ with the atomic one performed here. In the photon case the timing of the experiment is such that the small temporal width of the single-photon source ( $45 \mathrm{~ns})$, combined with a large interferometer $(48 \mathrm{~m})$, allow the choice event to be spacelike separated from the event where the photon reaches the first beamsplitter. In our experiment, the first beamsplitter is a light pulse which spatially overlaps the single-atom source, and thus the relevant temporal width for this operation is simply the duration of the $\pi / 2$ pulse $(20 \mu \mathrm{s})$. Thus space-like separation in our experiment is unfeasible; however, the slow velocity of the atom does allow a delayed choice in time, as implemented here.

As the data acquisition rates for a single-atom source are low (see Methods), we first demonstrate a delayed-choice interferometer with $\sim 1,000$ atoms per experimental cycle in the inset of Fig. 3 (where we plot the normalized probability of finding atoms in the $|0\rangle$ state), where the result for each choice of $\phi$ is the average of 20 experimental cycles. The distinction between the removal (blue points) and application (red points) of the mixing $\pi / 2$ pulse is very clear, as the former is $\sim 50 \%$ irrespective of the phase $\phi$, while the latter shows the expected sinusoidal dependence on $\phi$ typical of a Mach-Zehnder interferometer.

This experimental procedure is then reproduced with a true single-atom source ${ }^{16}$, and compared to the large-number result. The

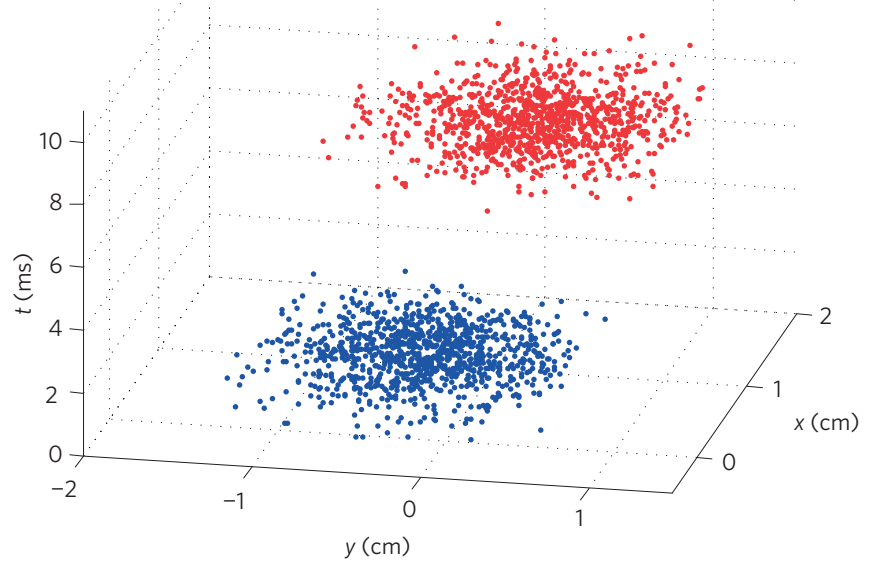

Figure $\mathbf{2}$ | Spatial and temporal locations of the output ports of the interferometer, showing the well-resolved detection locations. Blue data represent the counts observed at the $|0\rangle$ output port, whereas red data represent the $|1\rangle$ port. The atoms reaching port $|1\rangle$ arrive at a slightly latter time than those in port $|0\rangle$ due to the momentum kick they receive from the Bragg pulses.

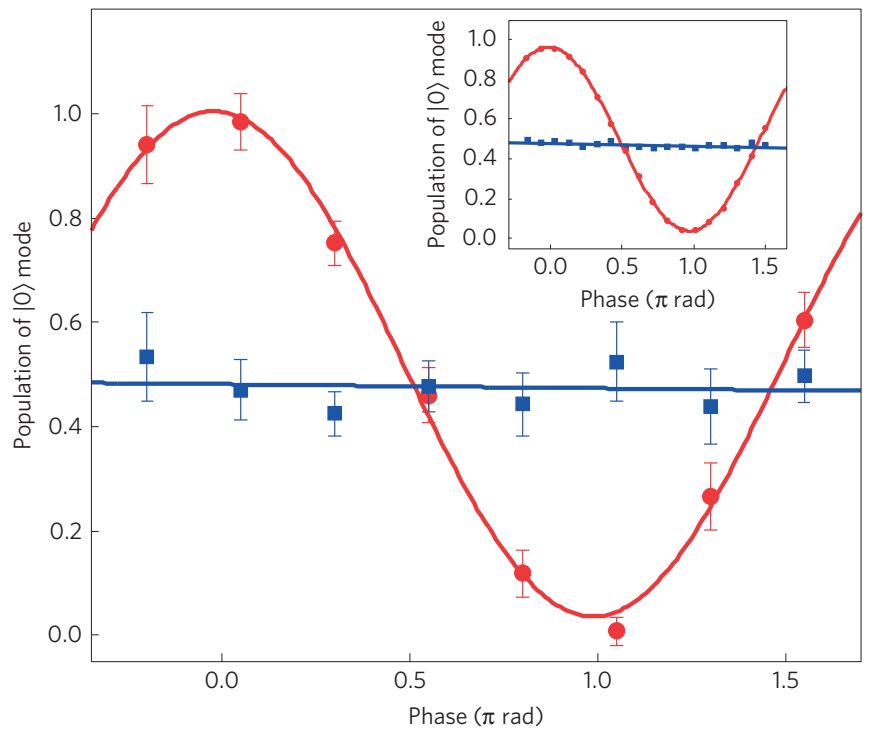

Figure 3 | Wheeler's delayed-choice experiment with massive bodies. Blue squares represent the open configuration and red dots represent the closed configuration of the interferometer. The inset shows the result when a large number of atoms $\sim 1,000$ are used, in such case the error bars ( 1 s.d.) are smaller than the data symbols used. The result shown in the main figure is produced using a single atom, with each point being the cumulative result of a few thousand runs of the experiment and the error bars representing the statistical spread of the data. The solid lines are fits to the data, with the closed configuration fitting well to a sinusoidal form with a visibility of $0.98 \pm 0.05$. A linear fit to the open configuration has a slight slope due to imperfections in our Bragg pulses.

result of this is shown in Fig. 3, where each experimental point is now the average of several thousand experimental cycles. It can be seen that the experimental points closely resemble that of Fig. 3 (inset), where fits to the experimental data give similar results to the large-number case. The main additional source of error for this data compared to the large-number version is shot noise due to limited counts. Also, the single-atom data are more susceptible to long-term 


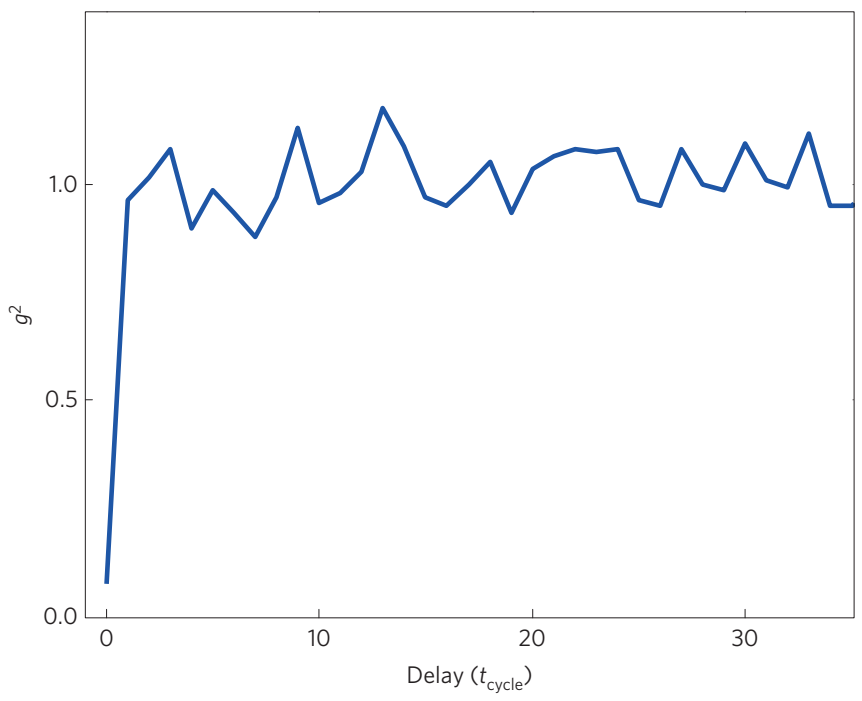

Figure 4 | Second-order correlation function of atoms arriving at the output ports of our interferometer as a function of the delay between experimental runs. Here the delay is in units of the cycle time of the experiment, where $t_{\text {cycle }}=35 \mathrm{~s}$.

drifts in the experiment, as data acquisition takes several weeks, as opposed to several hours for the large-number result.

We can quantify the single-atom nature of our source by analysing the statistics of atoms arriving at the output ports of the interferometer. To this end we calculate the second-order correlation function for the data shown in Fig. 3. We measure, see Fig. 4, a correlation function indicative of a strongly sub-Poissonian source, with a correlation parameter $\alpha$ (ref. 20) (equivalent to $g^{2}(0)$ ) of $\alpha=0.07 \pm 0.03$. The deviation we observe away from a perfect single-atom source $(\alpha=0)$ is readily explained via the measured dark count rate of our detector, with the probability of observing a real count and a dark count in the same run of the experiment, yielding a lower limit to $\alpha$ of 0.06 . This equates to observing, out of the nearly 30,000 runs of the experiment, ten runs in which two counts are observed.

As in the optical case ${ }^{2}$ we require each detector (see Fig. 2) to be unambiguously correlated to the path the atom took through the interferometer. To test this assumption, we use the 'whichway' parameter $I=\left(N_{1}-N_{2}\right) /\left(N_{1}+N_{2}\right)$ (refs 6,21,22), where $N_{1}$ and $N_{2}$ are the number of atoms measured at the output ports with the interferometer in the open configuration. We measure $I=0.97 \pm 0.05$ when the first beamsplitting pulse is absenteffectively allowing only one path through the interferometer. To check the other path we replace the first beamsplitting pulse with a mirror pulse, here we measure $I=0.95 \pm 0.05$, indicating that the ports of the interferometer allow excellent which-way information.

Further to this analysis, we can estimate the visibility when the interferometer is in the 'open' configuration by fitting a sinusoidal curve to the open data (blue squares in Fig. 3) and find a value of $V=0.03 \pm 0.04$. Consistent with theoretical predictions $^{6,23}$, and demonstrated for the more general intermediate case with photons ${ }^{24}$, we expect the complementarity parameter for our interferometer to follow the inequality: $V^{2}+D^{2} \leq 1$. For the open case we measure $V^{2}+D^{2}=0.92 \pm 0.08$, where $D$ is the distinguishability, given by the average of the whichway parameters. In the absence of information loss, one expects the complementarity parameter to be unity; in our case, the deviation from unity is attributable to imperfections in the Bragg pulses which reduce our distinguishability. The 'closed' configuration could be investigated in an analogous way by implementing the final beamsplitter, as could the intermediate case in which the Bragg beamsplitter pulse reflectivity is varied.

Wheeler's thought experiment is important since it tries to force a classical view of reality on to a quantum system. If one holds the view that to observe interference at the detector the photon must have traversed both arms (as a wave) of the interferometer (and conversely that the lack of interference unambiguously demonstrates the photon has traversed a single arm (as a particle)) then the 'delayed' choice creates a conundrum. In this picture, the choice of detection (delayed until after the photon has passed the first beamsplitter) is correlated with observing interference or no interference-and thus it seems that a future event (the method of detection) causes the photon to decide its past. If such a perspective seems untenable with a fast-moving massless photon, then our experiment, which uses a slow-moving massive helium atom (and thus is closer to our classical notions), makes this view of reality seem even more unlikely.

\section{Methods}

Methods and any associated references are available in the online version of the paper.

Received 9 October 2014; accepted 22 April 2015;

published online 25 May 2015; corrected online 3 June 2015

\section{References}

1. Wheeler, J. A. in Mathematical Foundations of Quantum Theory (ed. Marlow, A. R.) (Academic Press, 1978).

2. Jacques, V. et al. Experimental realization of Wheeler's delayed-choice gedanken experiment. Science 315, 966-968 (2007).

3. Bell, J. S. Speakable and Unspeakable in Quantum Mechanics (Cambridge Univ. Press, 1987).

4. de Broglie, L. Recherches sur la théorie des quanta. Ann. de Phys. 3, 22-128 (1925).

5. Davisson, C. \& Germer, L. H. Diffraction of electrons by a crystal of nickel. Phys. Rev. 30, 705-740 (1927).

6. Englert, B-G. Fringe visibility and which-way information: An inequality. Phys. Rev. Lett. 77, 2154-2157 (1996).

7. Ma, X., Kofler, J. \& Zeilinger, A. Delayed-choice gedanken experiments and their realizations. Preprint at http://arxiv.org/abs/1407.2930v1 (2014).

8. Hellmuth, T., Walther, H., Zajonc, A. \& Schleich, W. Delayed-choice experiments in quantum interference. Phys. Rev. A 35, 2532-2541 (1987).

9. Alley, C. O., Jacubowicz, O. G. \& Wickes, W. C. in Proceedings of the Second International Symposium on the Foundations of Quantum Mechanics (ed. Narani, H.) 36-47 (Physics Society of Japan, 1987).

10. Baldzuhn, J., Mohler, E. \& Martienssen, W. A wave-particle delayed-choice experiment with a single-photon state. Z. Phys. 77, 347-352 (1989).

11. Kim, Y-H., Yu, R., Kulik, S. P., Shih, Y. \& Scully, M. O. Delayed "choice" quantum eraser. Phys. Rev. Lett. 84, 1-5 (2000).

12. Ma, X-s. et al. Experimental delayed-choice entanglement swapping. Nature Phys. 8, 479-484 (2012).

13. Andrews, M. R. et al. Observation of interference between two Bose condensates. Science 275, 637-641 (1997).

14. Lawson-Daku, B. J. et al. Delayed choices in atom Stern-Gerlach interferometry. Phys. Rev. A 54, 5042-5047 (1996).

15. Kawai, T. et al. Realization of a delayed choice experiment using a multilayer cold neutron pulser. Nucl. Instrum. Methods Phys. Res. A 410, 259-263 (1998)

16. Manning, A. G., Khakimov, R., Dall, R. G. \& Truscott, A. G. A single atom source in the picokelvin regime. Phys. Rev. Lett. 113, 130403 (2014).

17. Martin, P. J., Oldaker, B. G., Miklich, A. H. \& Pritchard, D. E. Bragg scattering of atoms from a standing light wave. Phys. Rev. Lett. 60, 515-518 (1988).

18. Cronin, A., Schmiedmayer, J. \& Pritchard, D. Optics and interferometry with atoms and molecules. Rev. Mod. Phys. 81, 1051-1129 (2009).

19. Vassen, W. et al. Cold and trapped metastable noble gases. Rev. Mod. Phys. 84, 175-210 (2012).

20. Grangier, P., Roger, G. \& Aspect, A. Experimental evidence for a photon anticorrelation effect on a beam splitter: A new light on single-photon interferences. Europhys. Lett. 1, 173-179 (1986).

21. Durr, S., Nonn, T. \& Rempe, G. Fringe visibility and which-way information in an atom interferometer. Phys. Rev. Lett. 81, 5705-5709 (1998). 
22. Schwindt, P. D., Kwiat, P. G. \& Englert, B-G. Quantitative wave-particle duality and nonerasing quantum erasure. Phys. Rev. A 60, 4285-4290 (1999).

23. Jaeger, G., Shimony, A. \& Vaidman, L. Two interferometric complementarities. Phys. Rev. A 51, 54-67 (1995).

24. Jacques, V. et al. Delayed-choice test of quantum complementarity with interfering single photons. Phys. Rev. Lett. 100, 220402 (2008).

\section{Acknowledgements}

A.G.T. acknowledges the support of the Australian Research Council through the Future Fellowship grant FT100100468. The authors would like to acknowledge the technical assistance of C. Dedman as well as C. Savage for informative discussions.

\section{Author contributions}

A.G.M., R.G.D. and A.G.T. conceived the experiment. A.G.M. and R.I.K. performed the experiment and R.I.K. collected the data presented in this Letter. All authors contributed to the conceptual formulation of the physics, the interpretation of the data and writing the manuscript.

\section{Additional information}

Reprints and permissions information is available online at www.nature.com/reprints. Correspondence and requests for materials should be addressed to A.G.T.

\section{Competing financial interests}

The authors declare no competing financial interests. 


\section{Methods}

Source of ultracold $\mathrm{He}^{*}$. The experimental set-up is largely based on refs 25,26 . Ultracold $4 \mathrm{He}^{*}$ is magnetically trapped and evaporatively cooled ${ }^{27}$ to just below the Bose-Einstein condensate transition temperature $(\sim 1 \mu \mathrm{K})$. Approximately $10^{4}$ atoms are transferred into a vertical optical dipole trap by ramping up the intensity of a far red-detuned focused laser beam in the direction of gravity over $200 \mathrm{~ms}$. The magnetic trap is switched off and the only magnetic field present is generated by our magnetic field stabilization 'nullerometer ${ }^{28}$, which provides a bias magnetic field $\sim 1$ Gauss. Then, the dipole trap is ramped down over $100 \mathrm{~ms}$, resulting in a trap with harmonic frequencies of $\left(\omega_{x}, \omega_{y}, \omega_{z}\right) / 2 \pi=(1800,1800,12) \mathrm{Hz}$. The trap depth (equivalent to a temperature of a few nanokelvin) is then held at this point for two seconds, which allows the majority of the thermal atoms to exit the trap.

Single-atom source. To obtain a single-atom source ${ }^{16}$, we induce Penning ionization losses in the cloud described above by spin flipping the atoms from the $m_{j}=1$ state to the $m_{j}=0$ state with radio frequency (RF) radiation, which increases the rate of two-body Penning ionization loss by $\sim 5$ orders of magnitude ${ }^{29}$. The two-body collisional lifetime of $m_{j}=0$ atoms in our dipole trap is $(\sim 10 \mathrm{~ms})$, which is much shorter than the lifetime of $m_{j}=1$ atoms in the same trap $(\sim 25 \mathrm{~s})$ or the radiative lifetime of the metastable helium excited state $(7,870 \mathrm{~s} ;$ ref. 30$)$. By holding the $m_{j}=0$ atoms for $5 \mathrm{~s}$ we obtain a single atom in $50 \%$ of experimental cycles, and no atoms otherwise, depending on whether we start with an odd or even number of atoms in the trap, respectively. As each experimental cycle represents obtaining a single atom (or lack thereof), we are able to determine with confidence (with reference to detector efficiency $\sim 20 \%$ and trap lifetime) that we have only at most a single atom presen $t^{16}$. The atom is then released from the trap by switching off the dipole trapping beam.
Bragg interferometer. The Bragg beams are applied $\sim 1 \mathrm{~ms}$ after the atoms are released from the dipole trap and have a detuning $\sim 280 \mathrm{GHz}$ to the red of the $2^{1} \mathrm{~S}_{0}-2^{3} \mathrm{P}_{2}$ transition, and a relative detuning of $47 \mathrm{kHz}$ between the beams. The interferometer uses a $\pi / 2-\pi-\pi / 2$ pulse sequence, with each pulse being $20 \mu \mathrm{s}$ in duration and the $\pi$ pulse having $\sim 1.5$ times the intensity. The time between the beamsplitting pulses and the mirror pulse is $\sim 100 \mu$ s and a Quantis OEM quantum random bit generator (1D Quantique, model number: Quantis, P/N:0600684A210) is triggered and the resulting 'on' or 'off state is latched to an RF switch to randomly determine whether the second beamsplitting pulse is applied $70 \mu$ s after the mirror pulse finishes, which provides the 'delayed choice' for the experiment.

\section{References}

25. Dall, R. G. et al. Observation of atomic speckle and Hanbury Brown-Twiss correlations in guided matter waves. Nature Commun. 2, 291 (2011).

26. Dall, R. G. et al. Ideal $n$-body correlations with massive particles. Nature Phys. 9, 341-344 (2013).

27. Dall, R. G. \& Truscott, A. G. Bose-Einstein condensation of metastable helium in a bi-planar quadrupole Ioffe configuration trap. Opt. Commun. 270, 255-261 (2007).

28. Dedman, C. J., Dall, R. G., Byron, L. J. \& Truscott, A. G. Active cancellation of stray magnetic fields in a Bose-Einstein condensation experiment. Rev. Sci. Instrum. 78, 024703 (2007).

29. Shlyapnikov, G. V., Walraven, J. T. M., Rahmanov, U. M. \& Reynolds, M. W. Decay kinetics and Bose condensation in a gas of spin-polarized triplet helium. Phys. Rev. Lett. 73, 3247-3250 (1994).

30. Hodgman, S. S. et al. Metastable helium: A new determination of the longest atomic excited-state lifetime. Phys. Rev. Lett. 103, 053002 (2009). 


\section{CORRIGENDUM}

\section{Wheeler's delayed-choice gedanken experiment with a single atom}

A. G. Manning, R. I. Khakimov, R. G. Dall and A. G. Truscott

Nature Physics http://dx.doi.org/10.1038/nphys3343 (2015); published online 25 May 2015; corrected online 3 June 2015.

In the version of this Letter originally published, a sentence in the text describing the points in Fig. 3 was incorrect and should have read: 'The distinction between the removal (blue points) and application (red points) of the mixing $\pi / 2$ pulse is very clear, as the former is $\sim 50 \%$ irrespective of the phase $\phi$, while the latter shows the expected sinusoidal dependence on $\phi$ typical of a Mach-Zehnder interferometer.' This has now been corrected in all versions of the Letter. 